\title{
LA ENCAPSULACIÓN NOMINAL EN EL DISCURSO ACADÉMICO-CIENTÍFICO ORAL Y ESCRITO: PATRONES DE APARICIÓN*
}

\author{
NOMINAL ENCAPSULATION IN ORAL \\ AND WRITTEN ACADEMIC DISCOURSE: \\ GRAMMATICAL AND DISCOURSE PATTERNS
}

\author{
Anna López Samaniego \\ Universitat de Barcelona \\ alopezsam@ub.edu
}

Resumen: Este artículo analiza el funcionamiento de los encapsuladores nominales infieles en un corpus de discurso académico-científico en español formado por comunicaciones orales sobre lingüística presentadas en congresos y los artículos de investigación correspondientes publicados en revistas científicas o en actas de congreso (IO2 903 palabras). Se denomina encapsulador nominal infiel a aquel sustantivo que condensa el contenido de una o más predicaciones del discurso circundante y lo categoriza en tanto que entidad discursiva. Este mecanismo aparece habitualmente en patrones léxico-gramaticales como el patrón nominal especificativo (el hecho de que...), el patrón atributivo especificativo (el hecho es que...), o en patrones discursivos como el patrón atributivo anafórico, el patrón remático, el patrón temático y el patrón de marco interpretativo. Este trabajo se propone analizar las funciones y la frecuencia de aparición de estos patrones en dos modos de comunicación, oral y escrito. Los resultados del análisis muestran, de un lado, que la mayoría de estos patrones parecen ser más propios de alguna de estas dos modalidades y, además, que algunos encapsuladores presentan un grado considerable de atracción hacia ciertos patrones, lo cual constituye una prueba más de la estrecha relación existente entre léxico y gramática discursiva.

Palabras clave: encapsulador nominal, etiqueta discursiva, modo, oralidad, escritura, patrón discursivo, discurso académico-científico.

(*) Este estudio se enmarca en el proyecto interuniversitario EIIDA (Etude Interlinguistique et Interdisciplinaire des Discours Académiques), desarrollado entre los años 2012 y 20I5, y se ha realizado con el apoyo del laboratorio de excelencia Labex TransferS de la École Normale Supérieure de París (programa ANR-IOIDEX-0ooI-02 PSL y ANR-Io-LABX-oo99). 
Abstract: The following article discusses nominal unfaithful encapsulation on a corpus of Spanish conference presentations and the corresponding Proceedings articles in Linguistics (IO2 903 words). A nominal unfaithful encapsulator is a noun which sums up the content of a predicative portion of text and categorizes it as a discourse entity. This cohesive strategy is commonly used in lexico-grammatical patterns such as postnominal clause patterns (the fact that/to...), attributive complementing clause patterns (the fact is that/to...) or in discourse patterns such as anaphoric attributive pattern, rhematic pattern, thematic pattern and interpretative framework pattern. This study analyses the functions and frequency of these patterns in oral and written research genres. Results show that some of these patterns are more widely used in one of these two modes of communication. It is also argued that some of these encapsulators are attracted to some of these patterns, thus demonstrating the existence of close relationships between lexis and discourse grammar.

Key words: nominal encapsulation, discourse labels, mode, oral, written, discourse pattern, academic discourse.

$\cos \cos$

\section{ENCAPSULADORES NOMINALES INFIELES Y MODALIDAD COMU- NICATIVA}

El término encapsuladores nominales infieles se propone aquí para hacer referencia a un tipo de nombres de significado abstracto que, en el marco de sintagmas nominales definidos, condensan el contenido de una o más predicaciones del discurso circundante y lo categorizan en tanto que entidad discursiva. ${ }^{1}$ Son encapsuladores (término propuesto por Sinclair [1993] y empleado mayoritariamente por la tradición hispánica hasta la fecha) porque condensan o encapsulan el contenido de un segmento del discurso que suele incluir una o más predicaciones; además, se especifica que son nominales para diferenciarlos de otros mecanismos de encapsulación de significado aún menos específico de tipo pronominal (esto, ello). Por último, se toma aquí prestada la distinción propuesta por Apothéloz (1995) entre anáfora fiel e infiel, para especificar que se trata de mecanismos de encapsulación nominal que, además de condensar el

I. Aunque comparto la visión amplia del fenómeno que se sigue en otros estudios, que incluyen los usos exofóricos (Ivanic 199I; Flowerdew 2003), así como los encapsuladores que aparecen en sintagmas indefinidos o indeterminados (Ivanic I99I: II2; Benítez-Castro \& Thompson 2015: 380; Jiang \& Hyland 2015 y 2016), por motivos que se especifican en el apartado de metodología, opto aquí por la concepción más tradicional y aún mayoritaria de este mecanismo, que se limita a los empleos en sintagmas nominales definidos en los que la especificación del significado del nombre se lleva a cabo en el contexto discursivo. 
significado de un segmento textual, lo categorizan o le atribuyen una caracterización nueva como entidad del discurso (Schmid 2000: 13), como se observa en el ejemplo:

(I) Este autor opina que es «imposible reducir a un único diasistema las variedades del español» (Penny 2000: 6I). Es fácil ilustrar las razones de esta atinada afirmación.

Se trata, por tanto, del mecanismo que en otros trabajos se ha denominado etiquetas discursivas (López Samaniego 20II y 20I4) o, en el ámbito anglosajón, general nouns (Halliday \& Hasan 1976), anaphoric nouns (Francis 1986), shell nouns (Schmid 2000), signalling nouns (Flowerdew 2003) o metadiscursive nouns (Jiang \& Hyland 2016 y 20I7). Como se muestra en el ejemplo, el encapsulador nominal infiel atinada afirmación, además de condensar el contenido de la predicación anterior, lo categoriza o conceptualiza como una afirmación sostenida por el autor citado; además, en este caso, el encapsulador expresa incluso la actitud que adopta el autor del fragmento hacia dicha afirmación (es atinada, esto es, el autor está de acuerdo con ella). Como muestra el ejemplo, la función de estos encapsuladores nominales infieles es doble: de un lado, presentan una función textual cohesiva consistente en «poner en relación dos partes del texto que mantienen una relación de equivalencia (el miembro encapsulado y el encapsulador)» (López Samaniego 20I4: 267); de otro, desempeñan también una función pragmático-cognitiva, al «guiar al interlocutor sobre el modo en que debe interpretar el miembro encapsulado» (ibid.).

Entre los ámbitos discursivos en los que más se ha abordado el estudio de estas unidades destacan, en el ámbito anglosajón, géneros académicos como los artículos de investigación (Gray 20I0; Cortes 20I3; Jiang \& Hyland 20I5, 2016 y 2017), a veces en comparación con textos académicos escritos por estudiantes nativos o no nativos (Flowerdew 2006 y 20I0; Aktas \& Cortes 2008); los libros de texto (Tadros 1985, 1994); las tesis doctorales (Charles 2003, 2007), o incluso géneros orales que cubren desde conferencias hasta clases universitarias o conversaciones entre estudiantes, como el corpus MICASE compilado en la Universidad de Michigan (Swales 200I). ${ }^{2}$ En español, en cambio, los investigadores se han centrado en analizar el funcionamiento de este fenómeno en el discurso periodístico (Borreguero 2006, Llamas 20I0a y 20Iob; González Ruiz 20Io; Izquierdo \& González Ruiz 20I3b; López Samaniego 20I4) y político o, más concretamente, parlamentario (Izquierdo \& González Ruiz 2orza). En español, por tanto, el análisis de los encapsuladores nominales infieles se ha inclinado hacia

2. Una revisión mucho más exhaustiva, crítica y detallada de los artículos sobre encapsuladores nominales infieles en el discurso académico anglosajón puede leerse en Benítez-Castro y Thompson 20I5. 
el discurso profesional, con contadas excepciones de trabajos dedicados al discurso académico-científico (Moreno 2004; Álvarez de Mon 2006), generalmente centrados en el contraste entre la tradición retórica del español y del inglés.

Por otra parte, una dimensión en la que el análisis de los encapsuladores nominales se encuentra todavía en un estado incipiente es el contraste entre las modalidades escrita y oral de la comunicación. Algunos de los primeros trabajos que se han ocupado de comparar el empleo de estos mecanismos en ambos modos son los de Flowerdew (2003), Botley (2006), Korzen (2007), Yamasaki (2008), Flowerdew y Forest (2015). Estos trabajos, todos realizados sobre el inglés, han permitido identificar algunos empleos de los encapsuladores que parecen característicos del discurso oral, como la preferencia por aparecer en posición remática, así como la recurrencia de algunos patrones o colocaciones, como tener un problema o cometer un error (Yamasaki 2008: 90-9I). En cuanto a la frecuencia de aparición de encapsuladores nominales en general, si bien la mayoría de los estudios presuponen o concluyen a partir de corpus que es mayor en los géneros escritos (Flowerdew 2003; Korzen 2007), los análisis de géneros orales pertenecientes a lo que Ong (1982: 133) denomina «oralidad secundaria» (oral formal con cierto grado de planificación) demuestran que la frecuencia de los encapsuladores en géneros orales como el discurso parlamentario (Botley 2006: IO2) o la clase magistral (Flowerdew \& Forest 20I5: I00) puede llegar a equipararse a la que se observa en géneros escritos. ${ }^{3}$ Por último, autores como Flowerdew y Forest han identificado también algunas diferencias entre las modalidades oral y escrita en cuanto a la frecuencia de uso de determinados encapsuladores, especialmente constatando la preferencia de los textos orales por nombres de significado más general e inespecífico como cosa, punto, cuestión o idea (2015: IO3), tendencia ya apuntada por Swales (200I) que suele explicarse por el menor grado de planificación asociado a esta modalidad de comunicación.

El propósito de este trabajo es profundizar en el análisis de la variación de uso de los encapsuladores nominales en las modalidades oral y escrita en el ámbito académico-científico y, en concreto, en la caracterización de los patrones de aparición de este mecanismo. A través del análisis de textos orales y escritos pertenecientes a una disciplina poco atendida por los estudios sobre la encapsulación hasta el momento, la lingüística, este artículo se propone, de un lado, comprobar si puede defenderse también en español la preferencia de algunos patrones de aparición de este mecanismo discursivo por un determinado modo (oral o escrito); y, de otro lado, observar el

3. También se aprecia una misma frecuencia similar de aparición de los encapsuladores nominales infieles como mecanismo de mantenimiento referencial en textos orales y escritos en López Samaniego (20I7: 78), donde se analiza el mismo corpus manejado en el presente estudio. 
grado de atracción (attraction), en términos de Schmid (2000: 54-55), que los encapsuladores infieles presentan hacia determinados patrones gramaticales o discursivos en este tipo de discurso.

\section{METODOLOGÍA}

A fin de alcanzar los propósitos planteados, se ha analizado un corpus formado por diez comunicaciones de congreso y diez artículos de investigación derivados de dichas comunicaciones publicados en actas o en revistas especializadas. ${ }^{4}$ Las comunicaciones de congreso seleccionadas pertenecen a tres congresos: la Jornada io años de Filología Catalana en la UOC (Universitat Oberta de Catalunya, mayo de 2009), el Congreso Internacional de Pragmática del Español Hablado (Universitat de València, noviembre de 2009) y el XLI Simposio Internacional de la Sociedad Española de Lingüística (Universitat de València, enero-febrero de 20I2) y han sido transcritas empleando el software libre Transcriber I.5.I..$^{5}$

Ambos subcorpus, el oral y el escrito, son comparables por cuanto constituyen, de hecho, dos versiones de un mismo texto, ya que los artículos de investigación publicados en revistas o en actas constituyen versiones desarrolladas de las comunicaciones de congreso (Rowley-Jolivet 1999: 188). De este modo, puede considerarse que los dos géneros analizados pertenecen, por tanto, al mismo sistema de géneros, en términos de Bazerman (I994): el de los géneros de la investigación científica. Las comunicaciones de congreso presentan, además, similitudes con los textos propios del modo escrito, ya que son monológicas, altamente planificadas y a menudo se elaboran a partir de un borrador escrito.

En cuanto al tamaño, si bien ambos subcorpus están formados por el mismo número de textos (Io para cada género), no son fácilmente comparables en cuanto a número de palabras, ya que, como resulta esperable, el corpus escrito prácticamente

4. En los cuatro casos en los que no existían aún esas publicaciones derivadas, se han localizado artículos anteriores a la comunicación oral, elaborados por el mismo autor o autora y acerca del mismo tema.

5. La comunicación de la Jornada celebrada en la UOC está extraída de Youtube, mientras que las nueve comunicaciones restantes proceden de grabaciones consentidas facilitadas por las investigadoras Marta Albelda y María Estellés de la Universitat de València, a las que agradezco su ayuda y generosidad al facilitármelas. Mi agradecimiento se extiende, también, a los autores de las comunicaciones que muy amablemente me concedieron su consentimiento para utilizar sus textos. Por último, agradezco también al estudiante de doctorado Jorge Mauricio Molina Mejía, de la Université Stendhal - Grenoble III, su ayuda en la transcripción de tres de las comunicaciones que constituyen el corpus oral. Las publicaciones en actas y revistas analizadas no se consignan a fin de preservar el anonimato de los autores. 
dobla en extensión al oral, en una relación de 64427 palabras para el primero (una vez eliminados los agradecimientos, las tablas y figuras, los ejemplos y la bibliografía) por 38476 del segundo. No obstante, el desequilibrio en cuanto a la extensión de los corpus se salvará presentando los resultados como porcentajes. El conjunto del corpus analizado alcanza el tamańo medio de un corpus reducido estándar, en torno a las IOo ooo palabras (Ghadessy et alii 200I).

Dada la dificultad que suele comportar la identificación de los encapsuladores nominales en corpus reales, que suele requerir si no la búsqueda, al menos la revisión manual de los ejemplos, la metodología seguida para localizar los ejemplos, ha sido de tipo semi-automático, como resulta habitual en los estudios sobre estos mecanismos. Este método permite facilitar y agilizar la identificación automática de los ejemplos, así como garantizar la replicabilidad del estudio (Flowerdew \& Forest 2015: 8). Para poder llevar a cabo una primera búsqueda de forma automática, se ha optado por seleccionar únicamente los patrones abordados con mayor frecuencia por la bibliografía sobre el tema (establecidos por primera vez en Schmid 2000); a saber: los patrones discursivos que contienen sintagmas nominales introducidos por un determinante demostrativo (Charles 2003; Petch-Tyson 2000; Gray \& Cortes 20II), así como los patrones léxico-gramaticales de carácter nominal que entrañan estructuras apositivas, que aquí denomino patrón nominal especificativo (the fact that); los que aparecen en estructuras copulativas (Charles 2007; Schmid 2007; Jiang \& Hyland 2015), patrón atributivo especificativo (the truth is that); y los que contienen un demostrativo anafórico y un encapsulador nominal unidos por una relación atributiva, patrón atributivo anafórico (this is the truthla solid argument). La búsqueda se ha realizado utilizando el programa AntConc 3.4.4.o.

Para localizar ejemplos de los patrones discursivos con sintagmas nominales demostrativos, que comprenden los patrones atributivos anafóricos que acaban de mencionarse, se han realizado búsquedas para todas las formas de determinantes demostrativos, tanto las canónicas en español (este y ese, fundamentalmente, con sus variantes morfológicas correspondientes) como las de los determinantes dicho (de origen participial) y tal (de carácter cualitativo), que en español pueden funcionar con valor anafórico como variantes estilísticas de los demostrativos (Fernández Ramírez 195I: \$\$129 y I40; Mederos 1988: \$3.I6.I; Fernández Soriano 1999: \$14.3.5.3; RAE y ASALE 2009: \$r7.IO). Pese a que el uso de estas dos últimas formas es más restringido, se ha observado que algunos de los autores de los textos del corpus analizado las emplean de forma bastante sistemática.

Para identificar los patrones nominales especificativos (el hecho de que...), se han realizado las búsquedas <el/la/los/las \# de que $>$ y $<\mathrm{el} / \mathrm{la} / \mathrm{los} / \mathrm{las} \# \mathrm{de}{ }^{*} \mathrm{r}>$, a fin de 
localizar tanto los patrones complementados por oraciones completivas con verbo conjugado como los complementados por cláusulas de infinitivo (cf. ejemplos (2) y (3) de la tabla I, más adelante). También se han buscado ejemplos de esta estructura introducidos por determinantes posesivos, pero no se ha hallado ninguna ocurrencia. Por su parte, los patrones atributivos especificativos (el hecho es que...) se han localizado a partir de las siguientes búsquedas: <es/ha sido/fue/era/será/sería que $>\mathrm{y}<\mathrm{es} / \mathrm{ha}$ sido/fue/era/será/sería ${ }^{*} r>$, también para localizar aquellas estructuras que llevan como atributo tanto una oración sustantiva con verbo conjugado como una con infinitivo (cf. ejemplos (4) y (5), tabla I).

Una vez identificados automáticamente todos estos patrones y tras seleccionar manualmente solo aquellos casos en los que los nombres funcionaban como encapsuladores nominales infieles, ${ }^{6}$ todas las ocurrencias se han etiquetado mediante el programa Atlas.ti 7.5.7, siguiendo la clasificación de los patrones que se presenta en el siguiente apartado.

\section{PATRONES DE APARICIÓN DE LOS ENCAPSULADORES NOMINALES INFIELES}

A diferencia de otros estudios que han optado por este mismo método de búsqueda semi-automática, este estudio no se limita a una clasificación de los patrones de tipo únicamente léxico-gramatical, sino que tiene en cuenta también los ejemplos que contienen determinantes demostrativos de alcance discursivo. Estos últimos se clasificarán a partir de la función informativa del segmento en el que aparecen los encapsuladores nominales, siguiendo la propuesta presentada en López Samaniego (20I4) ${ }^{7}$

Así, en el presente trabajo, se distinguen tres patrones discursivos principales en los que se enmarcan las ocurrencias de $<$ demostrativo $+\mathrm{N}$ encapsulador $>$, estructura que arroja el mayor número de encapsuladores infieles (v. tabla i): (i) el patrón temático, en el que el nombre encapsulador aparece en la posición temática, ya sea por tematización o porque desempeña la función sintáctica de sujeto, ya sea como núcleo

6. Por ejemplo, en el caso de la búsqueda <es/ha sido/fuelera/será/sería ${ }^{*} r>$, ha sido necesario eliminar todas las ocurrencias de la partícula reformuladora es deciry los usos exofóricos de los sintagmas nominales demostrativos.

7. En este trabajo se opta por una simplificación de la propuesta presentada en López Samaniego (20I4), pues no interesa tanto atender a la diversidad de patrones discursivos en los que pueden aparecer estos encapsuladores como observar si estos tienen preferencia por una de las modalidades analizadas, tal como apunta Yamasaki (vid. \$I). 
del sintagma nominal o como complemento del nombre (ejemplo 7 de la tabla I); (ii) el patrón remático, en el que el nombre encapsulador aparece en segunda posición o de complemento (ejemplo 8); y el patrón de marco interpretativo, en el que el nombre aparece en una posición adelantada, en la periferia izquierda de la oración, a modo de predicación secundaria que condiciona la interpretación del enunciado al que se antepone (López Samaniego 20I4: 243) (ejemplo 9). Asimismo, se diferenciarán de los anteriores los casos en los que entre el demostrativo anafórico y el encapsulador aparece un verbo copulativo (patrón anafórico atributivo, ejemplo 6).

La tabla I sintetiza los patrones gramaticales y discursivos descritos hasta aquí y ofrece ejemplos de cada uno extraídos del corpus analizado:

\section{ORACIONALES}

Patrón nominal especificativo (La idea de que...):

$<$ Determinante $+\mathrm{EN}+\mathrm{de}+$ o. completiva $>$

(2) La evolución de excepto es muy similar / con la diferencia de que excepto no tenía la, tiene ya en latín únicamente el sentido exceptivo que tiene en castellano. (Оıо $)^{8}$

(3) Vamos a analizar estas señales en un ejemplo de conversaciones tomándolo como un tipo de interacción cara a cara con el objetivo de explicar un modelo de análisis del habla que integre señales verbales y no verbales desde una perspectiva en esencia pragmática de la lingüística. $\left(\mathrm{O}_{4}\right)$

Patrón atributivo especificativo (El hecho es que...):

$<$ Determinante $+\mathrm{EN}+\mathrm{v}$. ser + o.completiva $>$

(4) Mi experiencia es que se localiza sobre todo en / en el habla / en el discurso / es decir es un fenómeno que cuan- probablemente no llega a ser totalmente consciente en un hablante. (O6)

(5) Mi objetivo en esta comunicación sin embargo va / va a ser un poco distinto euh mi finalidad es analizar el funcionamiento que poseen aquellos elementos evidenciales que hacen referencia al receptor como garante de lo dicho. $\left(\mathrm{O}_{2}\right)$

8. En adelante, los ejemplos se identifican mediante una $\mathrm{O}$ o E que especifica el subcorpus citado (Oral o Escrito) seguida del número que identifica la comunicación o artículo (un mismo número identifica en ambos subcorpus una comunicación oral y el artículo derivado de esta). Asimismo, en los ejemplos los encapsuladores aparecen en negrita y el contenido encapsulado, subrayado. 
Anna López Samaniego

La encapsulación nominal en el discurso académico-cientifico oral y escrito: patrones de aparición

\section{DISCURSIVOS}

Patrón atributivo anafórico (Esta es la realidad):

$\{\text { MIEMBRO DISCURSO }\}^{\text {antecedente }}+\{$ Demostrativo + v. ser + determinante + EN $\}$

(6) Los indicadores implicados en la inversión de los principios de Manera e Informatividad, en especial con este último, crean efectos humorísticos en el texto. Este hecho permitiría localizar las relaciones entre ironía y humor en las ironías no prototípicas y, consecuentemente, explicar el humor a partir de la inversión de los principios de Manera e Informatividad. No obstante, este será el objetivo de investigaciones posteriores. (E3)

Patrón temático (Esta idea...):

$\{\text { MIEMBRO DISCURSO }\}^{\text {antecedente }}+\{[$ Tema: demostrativo + EN $]+[$ Rema $]\}$

(7) La transformación del castellano en español se presenta como un caso de la conversión de un dialecto en lengua: "el dialecto se va convirtiendo en lengua" (Alonso 1943: 54). Esta idea lleva a la concepción popular, alentada por muchos filólogos y lingüistas, de que la lengua es algo superior al dialecto. (EI)

Patrón remático (Smith interpreta esta idea como...):

$\{\text { MIEMBRO DISCURSO }\}^{\text {antecedente }}+\{[\text { Tema }]+[\text { Rema: demostrativo }+ \text { EN }]\}^{9}$

(8) El modelo relevantista, ampliamente aceptado por los investigadores, entiende la ironía como un eco de una proposición, que puede ser un enunciado dicho previamente o un pensamiento. [...]. Analicemos esta propuesta. $\left(\mathrm{E}_{3}\right)$

Patrón de marco interpretativo (En estas condiciones,...):

$\{\text { MIEMBRO DISCURSO }\}^{\text {antecedente }}+\{[$ Marco.: dem. + EN $]+[$ Rema $]\}$

(9) Dicho de otro modo: para que en la conversación coloquial española reine un clima comunicativo agradable, es necesario que los interlocutores que participan en ella posean un alto grado de confianza entre sí, y qué mejor manera de demostrarlo que pudiendo realizar una crítica sin que la otra persona se sienta ofendida. (...) En consecuencia, el elemento enunciativo sigue realzando el acto de emitir el enunciado, refuerza la asunción del dictum e intensifica lo dicho, pero, en esta situación comunicativa concretamarco, dicha intensificación atenúa el efecto social negativo de la crítica debido a que realza, precisamente, uno de los contenido de imagen básicos en ella: la confianza. (E2)

Tabla I. Patrones oracionales y discursivos de aparición de los encapsuladores nominales infieles (EN)

9. Los patrones temático y remático, así como el atributivo anafórico, se representan como patrones anafóricos porque esta es la dirección de la referencia en el $98 \%$ de los ejemplos examinados. Los casos en los que el encapsulador presenta un funcionamiento catafórico son muy escasos en el corpus examinado. 


\section{ANÁLISIS}

El resultado del análisis de los patrones de aparición de los encapsuladores nominales infieles en el corpus se sintetiza en la siguiente tabla, en la que se ofrece el número de ocurrencias de cada patrón, junto con el porcentaje que ese número representa sobre el total de encapsuladores nominales infieles identificados en cada subcorpus (entre paréntesis):

\begin{tabular}{lccc}
\hline Patrón & Comunicaciones orales & Publicaciones & Total \\
\hline Patrón nominal especificativo & $38(20,8 \%)$ & $91(31,6 \%)$ & 129 \\
\hline Patrón remático & $55(30,1 \%)$ & $58(20,1 \%)$ & 113 \\
\hline Patrón temático & $18(9,8 \%)$ & $75(26,1 \%)$ & 93 \\
\hline Patrón de marco interpretativo & $28(15,3 \%)$ & $51(17,7 \%)$ & 79 \\
\hline Patrón atributivo anafórico & $20(10,9 \%)$ & $7(2,4 \%)$ & 27 \\
\hline Patrón atributivo especificativo & $24(13,1 \%)$ & $6(2,1 \%)$ & 30 \\
\hline Total & 183 & 288 & 471 \\
\hline
\end{tabular}

Tabla 2. Patrones oracionales y discursivos identificados en el corpus

La tabla refleja, efectivamente, que la mayor parte de los patrones analizados aparecen de forma más recurrente en un determinado modo, a excepción del patrón de marco interpretativo, que presenta un índice de aparición muy similar en el subcorpus oral y en el escrito. En los epígrafes que siguen se desarrolla el análisis de los resultados para cada uno de los patrones clasificados.

\section{I EL PATRÓN NOMINAL ESPECIFICATIVO}

Uno de los patrones que parece presentar una preferencia clara por uno de los modos es el patrón nominal especificativo (el hecho de que...): casi tres cuartas partes de las ocurrencias de este patrón identificadas en el corpus (el 70 \%, 90 de los I29 casos) se han localizado en el subcorpus escrito. Además, este patrón oracional constituye el contexto de aparición más frecuente de los encapsuladores nominales infieles en los artículos de investigación analizados, dato que confirma la estrecha relación que parece existir entre este patrón y el discurso académico-científico escrito.

Una explicación de la mayor frecuencia de este patrón en textos escritos bien podría hallarse en su carácter catafórico. En este patrón nominal especificativo, el encapsulador funciona a modo de condicionante interpretativo del contenido encap- 
sulado (López Samaniego 20I4: 203), como mecanismo para orientar la interpretación del interlocutor sobre el discurso que sigue, como puede observarse en los siguientes ejemplos:

(Io) Cuando se habla de la unidad del español lo que se hace en realidad en muchos casos es interesarse por el mantenimiento del prestigio del espańol estándar de base castellana, pero induciendo en los demás la creencia de que los esfuerzos van encaminados a preservar el español como medio de comunicación neutral entre las diversas naciones hispanas. (EI)

(II) Nuestro estudio ha puesto de relieve la necesidad de diferenciar de manera tajante entre el plano verbal, la dimensión argumentativa y el efecto social en la determinación de las instrucciones pragmáticas de estos elementos. (E2)

En los ejemplos anteriores los encapsuladores destacados anticipan cómo debe interpretarse el contenido encapsulado en el contexto del discurso que se está elaborando y, en este sentido, resultan más eficaces y, esperablemente, más frecuentes cuando el emisor dispone de tiempo de planificación y revisión para seleccionar el sustantivo más preciso y más acorde con el sentido global del discurso que está construyendo como ocurre en el discurso escrito.

Entre los sustantivos que presentan cierto grado de atracción (Schmid 2007) hacia este patrón, pueden destacarse los nombres que expresan objetos o procesos mentales (sobre todo idea), nombres de significado metalingüístico relacionados con la comprensión del discurso (como sentido o interpretación) y, especialmente cuando la oración completiva va introducida por un infinitivo (II), sustantivos de significado modal como posibilidad o necesidad, en especial, con matiz volitivo (fin, intención, objetivo, pretensión). ${ }^{10}$ En la siguiente tabla se recogen los encapsuladores que se repiten en más de tres ocasiones en este patrón nominal especificativo en el corpus examinado, junto con grado de atracción que presentan hacia dicho patrón en cada uno de los subcorpus (porcentaje entre paréntesis): ${ }^{11}$

Io. Estos encapsuladores con matiz volitivo tienden a aparecer en estructuras semifijas con un valor conectivo de finalidad encabezadas por la preposición con (con el propósito / fin / objetivo de).

II. Este grado de atracción se ha calculado siguiendo la propuesta de Schmid (2007), que propone dividir la frecuencia del nombre en un patrón determinado (número de ocurrencias) entre la frecuencia total del patrón en un determinado corpus, y multiplicar el resultado por cien. 


\begin{tabular}{lccc}
\hline $\begin{array}{l}\text { Encapsulador } \\
\text { nominal infiel }\end{array}$ & $\begin{array}{c}\text { Ocurrencias y atracción en } \\
\text { el subcorpus oral }\end{array}$ & $\begin{array}{c}\text { Ocurrencias y atracción en } \\
\text { el subcorpus escrito }\end{array}$ & Total \\
\hline hecho & II $(29 \%)$ & I5 $(\mathrm{I} 6 \%)$ & $26(20 \%)$ \\
\hline idea & $3(8 \%)$ & IO (II $\%)$ & I3 $($ IO $\%)$ \\
\hline posibilidad & $4(\mathrm{IO} \%)$ & $8(9 \%)$ & I2 $(9 \%)$ \\
\hline sentido & $3(8 \%)$ & $5(5 \%)$ & $8(6 \%)$ \\
\hline caso & $3(8 \%)$ & $4(4 \%)$ & $7(3 \%)$ \\
\hline intención & $\mathrm{I}(2 \%)$ & $4(4 \%)$ & $5(2,5 \%)$ \\
\hline interpretación & - & $5(5 \%)$ & $5(2,5 \%)$ \\
\hline necesidad & - & $5(5 \%)$ & $5(2,5 \%)$ \\
\hline fin & - & $4(4 \%)$ & $4(2 \%)$ \\
\hline objetivo & I $(2 \%)$ & $3(3 \%)$ & $4(2 \%)$ \\
\hline acto & - & $4(4 \%)$ & $4(2 \%)$ \\
\hline
\end{tabular}

Tabla 3. Encapsuladores más frecuentes en el patrón nominal especificativo

Como se observa en la tabla, frente a los casos en los que el emisor parece seleccionar un encapsulador que le permita controlar la interpretación del discurso, resulta imposible soslayar las numerosas ocurrencias en las que se opta por un encapsulador de significado mucho más general que, sin embargo, presenta una elevada frecuencia en el corpus examinado: el sustantivo hecho. Si bien este encapsulador de significado general es el que más se repite en el patrón nominal especificativo en ambos subcorpus, ${ }^{12}$ se observa una mayor preferencia por encapsuladores más precisos en el subcorpus escrito, donde hecho aparece solo en el i6\% de las ocurrencias, que en el oral, donde el sustantivo hecho aparece en casi una tercera parte de los ejemplos del patrón identificados (casi el 30\% de las ocurrencias). Como ilustra el siguiente ejemplo, la presencia de este encapsulador aporta escaso contenido semántico y resulta totalmente prescindible:

(I2) En sí, nada tiene de extraño (et hecho de) que por y cierto se unan y den como resultado una locución adverbial de carácter epistémico, dada la naturaleza del segundo componente, cierto, que remite a la noción epistémica de 'certeza'. (E8)

Para explicar ejemplos como el de (I2), algunos autores han esgrimido motivaciones de tipo sintáctico, que aluden a la tendencia general de los hablantes a convertir oraciones sustantivas en sintagmas nominales, que es acusada tanto en español (Leonetti

I2. Este también parece ser el encapsulador más frecuente en este tipo de patrón en inglés (Schmid 2007). 
1999) como en otras lenguas tipológicamente alejadas como el inglés (Francis I993: 154). Sin embargo, parece plausible también que esta adecuación sintáctica en lenguas tipológicamente tan distintas vaya ligada a necesidades conceptuales y comunicativas: en concreto, a la necesidad de anclar como hecho real constatable una entidad compleja que en una oración sustantiva se conceptualizaría como proceso. Al encontrarse, además, bajo el alcance del artículo definido, el hecho queda anclado en la realidad y se presenta como familiar o conocido para los interlocutores; en definitiva, el hecho de opera a modo de anclaje conceptual (grounding, Langacker 2008: 259 y ss.), ${ }^{13}$ que convierte un proceso abstracto en un acontecimiento real y concreto.

\subsection{PATRONES DISCURSIVOS (TEMÁTICOS, REMÁTICOS Y DE MARCO INTERPRETATIVO)}

Tal como puede observarse en la Tabla 2, los sintagmas nominales demostrativos en posición temática o inicial, que los primeros trabajos sobre encapsuladores nominales habían identificado como lugar privilegiado de aparición de los encapsuladores nominales infieles (Halliday 1985/1994: 37 y ss.; Francis I994: 86-87; Schmid 2000: 330), constituyen el segundo patrón más frecuente de aparición de estos nombres en el subcorpus escrito (26,I\% del total). Los resultados obtenidos corroboran, pues, la tendencia de los encapsuladores a aparecer en posición inicial de oración indicada por gran parte de la bibliografía, pero solo en parte. Como ya se ha comentado, el patrón más frecuente en el subcorpus escrito no es el temático, sino el patrón nominal especificativo. Además, el predominio de los sintagmas nominales demostrativos con encapsuladores en posición temática sobre los que aparecen en posición remática se invierte en el subcorpus oral, en el que el patrón predominante es el que presenta los encapsuladores introducidos por demostrativos en posición remática. De hecho, en este subcorpus oral, el patrón remático triplica en frecuencia a los casos en los que los encapsuladores se encuentran en posición temática (30,1\% los primeros frente a 9,8\% los segundos).

Esta diferencia entre la posición informativa que ocupan los encapsuladores nominales en los géneros orales y escritos analizados, ya observada en Yamasaki (2008) para el inglés, podría reflejar una distinta función discursiva prioritaria de estos nombres en ambas modalidades. En el escrito, los encapsuladores tienden a emplearse en posición inicial, como mecanismo léxico de conexión entre oraciones o entre párrafos, en tanto que en el discurso oral se emplean más bien para colocar alguna

I3. Agradezco esta idea a uno de los revisores anónimos de este artículo. 
idea o concepto que ya ha aparecido en el contexto discursivo en el foco informativo (posición remática). En el ejemplo de (13) se ilustra la función más netamente cohesiva, orientada a engarzar oraciones y párrafos, que parecen desempeñar mayoritariamente los encapsuladores en las publicaciones científicas del subcorpus escrito examinado:

(I3) Rodríguez Rosique (2009) ha elaborado una propuesta de análisis sistemático del fenómeno en el que la ironía es una consecuencia de la violación explícita de la máxima de cualidad. La infracción de dicho requisito previo a la comunicación puede provocar la inversión, con carácter particular, de los principios conversacionales.

Esta fundamentación presenta, al menos, dos ventajas: en primer lugar, recoge bajo el concepto de inversión la idea tradicional de que la ironía es decir lo contrario de lo que dicen las palabras [...]. En segundo lugar, [...] relaciona la ironía con las inferencias que se atribuyen a ciertas formas lingüísticas. Dichos aspectos serán desarrollados en $\$$ III. (E3)

El primer encapsulador de este ejemplo se emplea para enlazar la fundamentación desarrollada en el primer párrafo con el análisis de esta que se lleva a cabo en el segundo, en tanto que el segundo (aspectos) recoge y etiqueta el contenido proposicional de la penúltima oración para introducir la última oración que, a su vez, anuncia el contenido de otro apartado del artículo. Resulta natural que la función cohesiva predomine en aquellos discursos (y modalidades comunicativas, como la escrita) que permiten un mayor grado de planificación, así como en los géneros en los que la estructuración del discurso resulta esencial para facilitar la comprensión, como los científico-académicos objeto del presente estudio.

Frente a la función cohesiva recurrente en las publicaciones del subcorpus escrito, los encapsuladores nominales infieles tienden a cumplir en el discurso oral una función más informativa o incluso evaluativa. En las comunicaciones de congreso analizadas, los encapsuladores nominales infieles se emplean a menudo para colocar en el foco informativo una idea o concepto que ya ha aparecido en el discurso (I4) o incluso, aunque no es lo más frecuente en este género, para introducir una evaluación explícita de esta (I5):

(I4) También creo yo que el pretérito simple puede ser resultativo // la cuestión está en que al emplear la forma compuesta el hablante quiere dejar claro el hecho de que la consecuencia perdura en el presente / (o)sea es un una manera enfática de marcar ese resultado. (O6)

(I5) [comentando un ejemplo] Bien pues lógicamente todos sabemos que los partidos en el poder tienen información privilegiada / lógicamente de la que carecen los otros // el e- denuncia / el locutor denuncia esta desigualdad (O5) 
Por lo que respecta a los tipos de nombres que suelen funcionar como encapsuladores en los patrones discursivos temático y remático en general, en la tabla 4 se recogen aquellos que presentan un mínimo de tres ocurrencias en alguno de los dos subcorpus:

\begin{tabular}{lccc}
\hline $\begin{array}{l}\text { Encapsulador } \\
\text { nominal infiel }\end{array}$ & $\begin{array}{c}\text { Ocurrencias y atracción } \\
\text { en el subcorpus escrito }\end{array}$ & $\begin{array}{c}\text { Ocurrencias y atracción } \\
\text { en el subcorpus oral }\end{array}$ & Total \\
\hline caso & $5(3,7 \%)$ & $2(2,4 \%)$ & $7(3, \mathrm{I} \%)$ \\
\hline uso & $2(\mathrm{I}, 5 \%)$ & $5(6 \%)$ & $7(3, \mathrm{I} \%)$ \\
\hline ejemplo & $4(3 \%)$ & $2(2,2 \%)$ & $6(2,6 \%)$ \\
\hline idea & $3(2, \mathrm{I} \%)$ & $3(3,6 \%)$ & $6(2,6 \%)$ \\
\hline afirmación & $3(2, \mathrm{I} \%)$ & $2(2,2 \%)$ & $5(2,2 \%)$ \\
\hline proceso & $3(2, \mathrm{I} \%)$ & $2(2,2 \%)$ & $5(2,2 \%)$ \\
\hline cuestión & $\mathrm{I}(\mathrm{O}, 7 \%)$ & $4(4,8 \%)$ & $5(2,2 \%)$ \\
\hline visión & $3(2, \mathrm{I} \%)$ & $\mathrm{I}(\mathrm{I}, 2 \%)$ & $3(\mathrm{I}, 8 \%)$ \\
\hline palabras & $3(2, \mathrm{I} \%)$ & $\mathrm{O} \%)$ \\
\hline hecho & $3(2, \mathrm{I} \%)$ & $\mathrm{O}$ & $3(\mathrm{I}, 3 \%)$ \\
\hline pasaje & $3(2, \mathrm{I} \%)$ & $\mathrm{O}$ & $3(\mathrm{I}, 3 \%)$ \\
\hline
\end{tabular}

Tabla 4. Encapsuladores más frecuentes en los patrones discursivos temático y remático

Tal como muestra la tabla, estos patrones discursivos no parecen tener preferencia por ningún encapsulador nominal en concreto, ya que el grado de atracción de todos los sustantivos que se repiten en alguna ocasión en el corpus no supera en prácticamente ningún caso el $5 \%$ del total de las ocurrencias. Por tanto, a diferencia de lo que ocurría con el patrón nominal especificativo, estos patrones discursivos se caracterizan por dar cabida a una gran variedad de encapsuladores léxicos infieles, si bien es cierto que muchos de los encapsuladores que se repiten pertenecen a un mismo tipo semántico: el de los nombres de significado lingüístico, que no etiquetan el contenido del segmento del discurso al que se refieren, sino que lo recuperan en tanto que segmento textual, como ocurre con palabras, pasaje, afirmación, ejemplo o uso (empleado en un sentido muy próximo al de ejemplo). La frecuencia de estos tipos de encapsuladores léxicos metalingüísticos debe atribuirse a la disciplina científicoacadémica seleccionada para el corpus, la lingüística, así como al tipo de razonamiento predominante en este ámbito de investigación: el argumento por el ejemplo (ejemplo que en este ámbito de especialidad suele ser un uso de la lengua concreto).

Cabe mencionar que este empleo recurrente de encapsuladores que aluden al valor ilocutivo (generalmente, ilustrador) de un fragmento textual previo afecta también 
a un encapsulador de significado muy inespecífico: el sustantivo caso que, además de ser el más frecuente en los patrones temáticos y remáticos (cf. tabla 4) presenta un grado de atracción muy elevado hacia el patrón discursivo de marco interpretativo. En concreto, este sustantivo copa casi la mitad de los encapsuladores seleccionados en este patrón ( 37 de las 83 ocurrencias). En la mayor parte de las ocurrencias de caso en el patrón de marco interpretativo en el corpus examinado, se emplea en el contexto del comentario de algún ejemplo, como ocurre en (I6), donde el sustantivo encapsula el ejemplo subrayado y lo presenta como realización individual o instancia lingüística (enunciado) para la que es válida la afirmación que sigue:

(I6) En el primer ejemplo / ¿está ya la comida? No, bajamos, bajamos a ver si está / en este caso el significado comprobativo que encontramos en la estructura independiente también aparece en la estructura dependiente. (Oıо)

El sustantivo caso se emplea también, especialmente en el subcorpus escrito, para referirse a otro tipo de situaciones no comunicativas, en términos más generales:

(I7) Cuando el elemento enunciativo se utiliza al inicio de respuesta en una situación de no enfrentamiento, el emisor, gracias a su uso, dispone de tiempo para pensar y organizar su intervención, y, además, indica que está dispuesto a ser colaborativo y cooperativo.

El objetivo del emisor es, en este caso, fomentar la empatía con el receptor. $\left(E_{2}\right)^{14}$ En ambos ejemplos, la aparición del encapsulador caso se explica por la capacidad de este sustantivo para referirse en términos generales al contexto, situación o instancia específica a los que se limita la validez de una afirmación, significado que encaja perfectamente con la función informativa propia del patrón discursivo de marco interpretativo, consistente en acotar o circunscribir la validez de la predicación principal a un determinado contexto o situación (Gutiérrez Ordóńez 1997: 40). En el discurso académico-científico en concreto, esta función informativa permite, además, desempeñar una función discursiva esencial en este tipo de discurso: la de atenuador (hedge en Hyland 1998) restrictivo, que limita la validez de la afirmación a una situación o contexto específico; tal contexto es el ejemplo citado, en (I6) y la situación comunicativa descrita en el segmento subrayado, en el ejemplo de (I7).

I4. Tal como acertadamente me comenta uno de los revisores anónimos de este estudio, contextos como el de (I6), en los que caso se emplea para hacer referencia a una instancia concreta, podrían concebirse como contextos-puente hacia usos incidentales más gramaticalizados como el de (I7) —en el que el sustantivo no se refiere ya tan claramente a una situación o cosa concreta, sino a un tipo de situaciones_-, o incluso hacia empleos de en este caso en los que el significado del sustantivo se diluye aún más en la función atenuadora de la expresión. 


\section{$4 \cdot 3$ PATRONES ATRIBUTIVOS}

La tabla 2 muestra también diferencias de interés en el comportamiento de los patrones atributivos anafórico (esta es la verdad) y especificativo (la verdad es que...) en los subcorpus comparados. En primer lugar, cabe indicar que estos patrones son los menos frecuentes en ambos subcorpus. Esta baja frecuencia, por tanto, debería explicarse a partir de algún rasgo que ambos géneros tengan en común como, por ejemplo, el hecho de tratarse de textos formales con un cierto grado de planificación. De hecho, la frecuencia de estos patrones es mucho menor en el subcorpus escrito, que puede considerarse más formal por su mayor grado de planificación y revisión, que en el oral. Como puede deducirse a partir de la tabla, más de tres cuartas partes de los patrones atributivos identificados en el corpus se han localizado en el subcorpus oral (44 ocurrencias del total de 57). Estas cifras apuntan a una preferencia clara de estos patrones atributivos por la modalidad oral, que podría venir determinada, como se ha comentado, por el menor grado de formalidad y de planificación que esta presenta, incluso en géneros no prototípicos como las comunicaciones de congreso, en los que el discurso oral se aproxima al estilo comunicativo propio de la escritura. ${ }^{15}$

Junto al grado de formalidad y planificación menor que presentan las comunicaciones orales, otro factor que puede explicar la mayor frecuencia de estas estructuras en el subcorpus oral es la función focalizadora que desempeñan. Tal como han indicado autores como Schmid, el patrón atributivo especificativo permite al emisor situar toda una predicación, el contenido encapsulado, en la posición remática propia del foco informativo y, además, llamar la atención sobre ella mediante el encapsulador que aparece en posición temática, que anuncia catafóricamente su contenido (2000: 332 y 200I: I535), como se observa en el fragmento subrayado del ejemplo de (I8):

(I8) [El motivo de la elección de estas señales de la risa y la sonrisa ${ }^{\text {TEMA }}$ es que ambas pueden considerarse a causa de sus características intrínsecas o sea acerca de sus características como señales en sí mismas. $\left(\mathrm{O}_{4}\right)$

I5. Si bien este comportamiento de los patrones atributivos se ha observado en un corpus reducido, confirma los resultados obtenidos por Flowerdew y Forest (2015) para el inglés. Más de la mitad de los ejemplos correspondientes al patrón atributivo especificativo que estos autores identifican en su corpus de textos académicos se encuentran en clases magistrales, el único género oral incluido en su corpus, con un grado de formalidad esperablemente menor que el de nuestras comunicaciones de congreso.

Caplletra 64 (Primavera, 2018), pp. 129-152 
Por su parte, el patrón atributivo anafórico también puede considerarse una construcción con función focalizadora. ${ }^{16}$ Este patrón permite colocar el encapsulador nominal en la posición remática o de foco informativo y llamar la atención sobre el contenido encapsulado gracias a su repetición mediante el pronombre demostrativo, como ocurre en el siguiente ejemplo:

(19) No las podemos estudiar ni a estas señales ni a otras señales/ ya sean verbales o no verbales / desde el punto de vista del mensaje como recursos aislados / sino integradas a la significación del texto entendiendo por texto a un conjunto congruente con el contexto coherente con el cotexto interno y socialmente adecuado en relación a la relación interpersonal que se desarrolla en un discurso // euh entonces lo / este es el / el el digamos la definición de texto en la que queremos incorporar a los no verbales. $\left(\mathrm{O}_{4}\right)$

El patrón atributivo anafórico se emplea en (19) para destacar la idea de que la expuesta (y no otra) es la definición de texto relevante para comprender el argumento que se pretende defender. El emisor emplea una estructura que focaliza un segmento del discurso (la definición de texto encapsulada, frente a otras alternativas posibles) para insistir en la idea de que el contenido encapsulado es la definición de texto que interesa al emisor y destacar la importancia de ese contenido encapsulado en su exposición. Además, este patrón, a diferencia del anterior, permite que tanto la definición en sí como el encapsulador destacado en negrita aparezcan en posiciones de foco informativo, ya que se encuentran en oraciones distintas.

Por último, otro rasgo que puede incidir en la mayor frecuencia de los dos patrones atributivos en el discurso oral es la función evaluativa que permiten desempeñar. Tal como ha indicado la bibliografía, el patrón discursivo anafórico permite evaluar el contenido proposicional encapsulado de manera explícita (Yamasaki 2008: 79), al colocar el encapsulador en una posición de foco informativo, en tanto que en el nominal especificativo tal evaluación o caracterización se da de manera implícita o presupuesta, logrando lo que Schmid ha descrito como un «efecto objetivador» (200I: I539-I540). Los ejemplos que siguen ilustran el carácter evaluativo explícito (22) o implícito (2O-2I) de estos patrones, que pueden presentar tanto valoraciones subjetivas del emisor (20), como valoraciones de tipo epistémico (2I-22):

I6. Se trata de focalizaciones en sentido diferente: el empleado en el ámbito anglosajón, consistente en colocar una información en el foco informativo, es decir, en posición remática, para los patrones atributivos especificativos y, por otro lado, la focalización que consiste en llamar la atención del interlocutor sobre un determinado segmento del discurso, como ocurre, a mi modo de ver, con el encapsulador infiel en los patrones atributivos anafóricos. 
(20) En el caso de a ver si hay una serie de restricciones sintácticas // el problema es que $a$ ver si claramente se identifica como una construcción y no tanto otros elementos que se han gramaticalizado como el caso de los futuros románicos. $\left(\mathrm{O}_{9}\right)$

(2I) Otra posibilidad es que el castellano sea más fácil de entender que el catalán / lo cual es un absurdo total. (OI)

(22) Es decir tiene un camino de evolución totalmente distinto del de los marcadores que entran por convencionalización de inferencias / y podríamos decir que es una especie de lexicalizaciones pragmáticas / es decir que lo que vamos a esperar es que se queden un poco arrinconados / que se queden arrinconados en el registro culto de la lengua [...] ]// esa sería la predicción o las predicciones que proponemos para los elementos que se creen a partir de este tipo de gramaticalizaciones por tradiciones discursivas. $\left(\mathrm{O}_{7}\right)$

En el ámbito académico-científico, la expresión más o menos velada de la actitud del hablante sobre el contenido de su discurso parece presentarse, en general, con menor frecuencia en los géneros escritos que en los orales, que, por su mayor provisionalidad, tienden a presentar la información de forma menos asertiva y a incluir más valoraciones de determinados aspectos del estudio (Rowley-Jolivet 1999: I88 y 20I2: I62). Es de esperar, por tanto, que estructuras evaluativas como los patrones atributivos resulten más frecuentes en la modalidad oral.

En cuanto a los nombres que presentan una mayor frecuencia en estos patrones, lo cierto es que el número escaso de ocurrencias localizadas en el corpus no permite identificar tendencias generales, aunque sí apunta a la probabilidad de que algún tipo de nombres resulten más frecuentes. Así, entre los nombres que se repiten en más de tres ocasiones en ambos patrones se encuentran caso (4 ocurrencias) en el patrón anafórico y problema (4) en el patrón especificativo.

\section{CONCLUSIONES}

Los datos obtenidos a partir del corpus examinado, junto con el análisis cuantitativo y cualitativo llevado a cabo, indican que los patrones de aparición de los encapsuladores nominales infieles dependen considerablemente del modo o modalidad comunicativa del discurso. Se han observado diferencias de frecuencia relevantes entre ambas modalidades en todos los patrones oracionales y discursivos examinados, a excepción del patrón discursivo de marco interpretativo, que presenta una frecuencia similar en ambos subcorpus. Para justificar estas diferencias, y también la semejanza entre los dos subcorpus, se ha partido de la función que los patrones desempeñan en el discurso. Así, por ejemplo, el índice de frecuencia similar que presenta el patrón

Caplletra 64 (Primavera, 2018), pp. 129-152 
de marco interpretativo en ambos subcorpus refleja que este patrón desempeńa una función recurrente en el ámbito discursivo y en los géneros concretos seleccionados: la de atenuador que limita la validez de la afirmación que sigue a una situación o contexto específico. Esta función discursiva es característica del discurso científico, en el que a menudo es relevante destacar la provisionalidad del conocimiento.

En cuanto a las diferencias entre ambas modalidades, destaca, de un lado, el patrón nominal especificativo, que es mucho más frecuente en las publicaciones científicas que en las comunicaciones orales, y de otro, los patrones atributivos, tanto especificativos como anafóricos, en los que ocurre a la inversa. En el primero, la preferencia por el modo escrito podría deberse a motivaciones tanto pragmáticas como sintácticas, ya que se emplea tanto para guiar o condicionar la interpretación del discurso en textos planificados como, en algunos casos, para convertir oraciones sustantivas en sintagmas nominales. En el caso de los patrones atributivos, su mayor frecuencia en el discurso oral podría explicarse tanto por la función informativa focalizadora que ambos desempeñan como por la función evaluadora explícita o implícita que tienden a realizar. La frecuencia baja, en general, de estos últimos patrones en ambos subcorpus podría reflejar también una cierta preferencia por registros menos formales, aunque esta hipótesis debería confirmarse a partir de la ampliación del estudio a corpus formados por textos más informales.

Por último, el análisis confirma la tendencia de los encapsuladores nominales a aparecer en posición remática en el discurso oral apuntada por Yamasaki (2008). En estudios futuros puede resultar de gran interés contrastar los resultados obtenidos aquí con otros géneros orales más prototípicos, como la conversación o incluso la clase magistral, a fin de poder observar si el discurso oral de la investigación científica se aproxima más a la prosa científica o a la oralidad prototípica, en la línea de otros estudios que se han llevado a cabo para la lengua inglesa (Swales 1990; CarterThomas \& Rowley-Jolivet 200I).

Por lo que respecta a la relación entre estos patrones y determinados tipos de encapsuladores, pese a que el tamaño reducido del corpus no permite alcanzar conclusiones categóricas, cabe destacar por su elevada frecuencia dos encapsuladores: caso y hecho. Además de resultar muy recurrentes, lo más interesante es que aparecen ligados a dos patrones concretos: el patrón de marco interpretativo y el patrón nominal especificativo, respectivamente. En este sentido, el presente estudio ha mostrado el interés que reviste el análisis de la relación entre los patrones de aparición de los encapsuladores nominales y la selección léxica de estos nombres. De nuevo, resultará de gran interés poder corroborar y ampliar la especialización de estos y probablemente otros encapsuladores aplicando la metodología de este estudio a otros tipos de texto y ámbitos discursivos. 


\section{REFERENCIAS BIBLIOGRÁFICAS}

Aktas, R. N. \& V. Cortes (2008) «Shell nouns as cohesive devices in published and ESL student writing», Journal of English for Academic Purposes, 7 (I), pp. 3-I4.

Álvarez De MON, I. (2006) «A contrastive study of encapsulation and prospection in written scientific text», en J. Flowerdew \& M. Gotti (ed.), Studies in specialized discourse), Berna, Peter Lang, pp. 2I-40.

AротнÉLOz, D. (1995) Rôle et fonctionnement de l'anaphore dans la dynamique textuelle, Genève, Droz.

BAZERMAN, C. (1994) «Systems of genres and the enactment of social intentions», en A. Freedman \& P. Medway (ed.), Genre and the new rhetoric, London/Bristol, Taylor \& Francis, pp. 79-IOI.

Benítez-Castro, M. Á. \& P. Thompson (20I5) «Shell-nounhood in academic discourse: A critical state-of-the-art review», International Journal of Corpus Linguistics, 20 (3), pp. 378-404.

Borreguero Zuloaga, M. (2006) «Naturaleza y función de los encapsuladores en los textos informativamente densos (la noticia periodística)», Cuadernos de Filología Italiana, 13, pp. 73-95.

BotLey, S. P. (2006) «Indirect anaphora: Testing the limits of corpus-based linguistics», International Journal of Corpus Linguistics, II (I), pp. 73-II2. [<http://doi. org/IO.IO75/ijcl.II.I.04bot>]

Carter-Thomas, S. \& E. Rowley-Jolivet (200I) «Syntactic differences in oral and written scientific discourse: the role of information structure», $A S p$, 33 (3I-33), pp. 19-37. [<http://doi.org/I0.400o/asp.I752>]

Charles, M. (2003) “"This mystery...”: a corpus-based study of the use of nouns to construct stance in theses from two contrasting disciplines", Journal of English for Academic Purposes, 2, pp. 313-326.

- (2007) «Argument or evidence? Disciplinary variation in the use of the Noun that pattern in stance construction", English for Specific Purposes, 26 (2), pp. 203-218. [<http://doi.org/Io.Ior6/j.esp.2006.08.004>]

Contes, V. (2013) «The purpose of this study is to: Connecting lexical bundles to moves in research article introductions", Journal of English for Academic Purposes, I2 (I), pp. 33-43.

Fernández Ramírez, S. (I95I) Gramática española. 3.2. El pronombre, Madrid, Arco Libros, vol. 2.

Fernández Soriano, O. (1999) «El pronombre personal. Formas y distribuciones. Pronombres átonos y tónicos», en I. Bosque \& V. Demonte (eds.),

Caplletra 64 (Primavera, 2018), pp. 129-152 
Gramática Descriptiva de la Lengua Española, Madrid, Espasa Calpe, vol. I, pp. I209-I274.

FLOWERDEW, J. (2003) «Register-specificity of signalling nouns in discourse», en P. Leistyna \& C. F. Meyer (ed.), Corpus Analysis: Language Structure and Language Use, Amsterdam / New York, Rodopi, pp. 35-46.

- (2006) «Use of signalling nouns in a learner corpus», International Journal of Corpus Linguistics, II (3), pp. 345-362.

- (2010) "Use of signalling nouns across Li and L2 writer corpora», International Journal of Corpus Linguistics, $\mathrm{I} 5$ (I), pp. 36-55.

Flowerdew, J. \& R. W. Forest (20I5) Signalling nouns in English, Cambridge, Cambridge University Press.

Francis, G. (1986) Anaphoric nouns, Birmingham, English Language Research.

- (1993) «A Corpus-Driven Approach to Grammar. Principles, Methods and Examples», en M. Baker, G. Francis y E. Tognini-Bonelli (eds.), Text and Tachnology, In honour of John Sinclair, Amsterdam/Philadelphia, John Benjamins, pp. I37-I56.

- (1994) "Labelling discourse: an aspect of nominal-group lexical cohesion», M. Coulthard (ed.), Advances in written text analysis, London / New York, Routledge, pp. 83-IOI.

Ghadessy, M., A. Henry \& R. Roseberry, ed. (200I) Small Corpus Studies and ELT: Theory and Practice, Amsterdam, John Benjamins.

GonzÁlez RuIZ, R. (20IO) «Gramática y discurso: nominalización y construcción discursiva en las noticias periodísticas», en C. Martínez Pasamar (ed.), Estrategias argumentativas en el discurso periodistico, Frankfurt am Main, Peter Lang. pp. II9-I46.

GraY, B. (20I0) «On the use of demonstrative pronouns and determiners as cohesive devices: A focus on sentence-initial this/these in academic prose», Journal of English for Academic Purposes, 9, I67-183.

Gutiérrez OrdóńEz, S. (1997) Temas, remas, focos, tópicos y comentarios, Madrid, Arco Libros.

Halliday, M. A. K. (1985) An introduction to functional grammar, London, Arnold London.

Halliday, M. A. K. \& R. Hasan (1976) Cohesion in English, London, Longman.

IVANIČ, R. (I99I) "Nouns in search of a context: a study of nouns with both openand closed-system characteristics», International Review of Applied Linguistics in Language Teaching, 29 (2), pp. 93-II4.

IzQuierdo, D. \& R. GonzÁLEZ Ruiz (2013a) «Encapsulación y etiquetas discursivas en el discurso parlamentario: función argumentativa a partir de un corpus paralelo", Oralia, 16, pp. 209-243. 
- (2013b) «La evaluación a través de etiquetas discursivas en un género de opinión: análisis de un corpus de editoriales de $A B C$ y El Pais», en C. Llamas Saíz, C. Martínez Pasamar \& M. Casado Velarde (ed.), Léxico y argumentación en el discurso público actual, Frankfurt, Peter Lang, pp. I49-185.

JiAnG, F. \& K. Hyland (20I5) “The fact that': Stance nouns in disciplinary writing», Discourse Studies, I7 (5), pp. 529-550.

- (2016) «Nouns and Academic Interactions: A Neglected Feature of Metadiscourse», Applied Linguistics, pp. I-25. [<http://doi.org/I0.I093/applin/amw023>]

- (2017) «Metadiscursive nouns: Interaction and cohesion in abstract moves», English for Specific Purposes, 46 (November 20I6), pp. I-I4. [<http://doi.org/IO.IoI6/j. esp.2016.II.OOI>]

Korzen, I. (2007) «Linguistic typology, text structure and anaphors», en I. Korzen \& L. Lundquist (eds.), Comparing Anaphors Between Sentences, Texts and Languages, Copenhague, Samfundslitteratur Press, pp. 93-I09.

Langacker, R. W. (2008) Cognitive Grammar. A basic introduction, New York, Oxford University Press.

Leonetti, M. (1999) «El artículo», en I. Bosque \& V. Demonte (dir.), Gramática descriptiva de la lengua española, Madrid, Espasa, vol. I, pp. 787-890.

Llamas, C. (20IOa) «Argumentación en la noticia periodística: el caso de la anáfora conceptual metafórica», en C. Martínez Pasamar (ed.), Estrategias argumentativas en el discurso periodístico, Frankfurt am Main, Peter Lang, pp. I47-170.

LLAMAS, C. (20IOb) «Interpretación del discurso ajeno: la anáfora conceptual metafórica en la noticia periodística», Revista de Investigación Lingüistica, I3, pp. I07-I26.

López Samaniego, A. (20iI) «La categorización de entidades del discurso en la escritura profesional. Las etiquetas discursivas como mecanismo de cohesión léxica», Barcelona, Universitat de Barcelona, tesis doctoral. [<http://hdl.handle. net/I0803/48757>, fecha de consulta: I/5/2017.]

López SAMANIEgo, A. (20I4) Las etiquetas discursivas: cohesión anafóricay categorización de entidades del discurso, Pamplona, EUNSA.

- (2017) «Deixis textual y discursiva en el discurso científico-académico oral y escrito», CHIMERA: Revista de Corpus de Lenguas Romances y Estudios Lingüisticos, 4.I, pp. 6I-88.

Mederos, H. (1988) Procedimientos de cohesión en el español actual, Santa Cruz de Tenerife, Publicaciones Científicas del Excelentísimo Cabildo Insular de Tenerife. Moreno, A. I. (2004) «Retrospective labelling in premise-conclusion metatext: an English-Spanish contrastive study of research articles on business and economics", Journal of English for Academic Purposes, 3 (4), pp. 32I-339. 
Real Academia Española y Asociación de Academias de la Lengua Española (2009) Nueva gramática de la lengua española, Madrid, Espasa-Calpe.

Rowley-Jolivet, E. (I999) "The pivotal role of conference papers in the network of scientific communication", $A S p$, 26, pp. 179-196. [<http://ww.w.asp.revues. org/2394>, fecha de consulta $\mathrm{I} / 5 / 2017$.]

- (20I2) «Oralising text slides in scientific conference presentacions», en A. E. Boulton, S. Carter-Thomas \& E. Rowley-Jolivet (ed.), Corpus-Informed Research and Learning in ESP. Issues and applications, Bern, John Benjamins, pp. I37-I65. [<http://doi.org/IO.IO75/sll.I5.I.07mor > ]

Schmid, H.-J. (2000) English abstract nouns as conceptual shells: From corpus to cognition, Berlin / New York, Walter de Gruyter.

- (200I) “"Presupposition can be a bluff": How abstract nouns can be used as presupposition triggers», Journal of Pragmatics, 33 (IO), pp. I529-I552.

- (2007) «Non-compositionality and emergent meaning of lexico-grammatical chunks: A corpus study of noun phrases with sentential complements as constructions", Proceedings of the I6th International Congress of Linguists (Paris, July 20-25, I997), 55 (3), pp. 313-340.

Sinclair, J. M. (I993) «Written discourse structure», en J. M. Sinclair, M. Hoey \& G. Fox (ed.), Techniques of description: Spoken and Written Discourse, London / New York, Routledge, pp. 6-3I.

Swales, J. (I990) Genre analysis: English in academic and research settings, New York / Cambridge, Cambridge University Press.

— (200I) "Metatalk in American academic talk: The cases of point and thing», Journal of English Linguistics, 29 (I), pp. 34-54.

Tadros, A. (1985) Prediction in text, Birmingham, University of Birmingham.

- (1994) "Predictive categories in expository text», en M. Coulthard (ed.), Advances in written text analysis, London / New York, Routledge, pp. 69-82.

Yamasaki, N. (2008) «Collocations and colligations associated with discourse functions of unspecific anaphoric nouns», International Journal of Corpus Linguistics, I3(I), pp. 75-98. 